\title{
Malaria outbreak investigation and contracting factors in Simada District, Northwest Ethiopia: a case-control study
}

\author{
Baymot Workineh ${ }^{1}$, Fantahun Ayenew Mekonnen ${ }^{1}$, Mekonnen Sisay ${ }^{2 *}$ and Kedir Abdela Gonete ${ }^{2}$
}

\begin{abstract}
Objective: The aim of this study was to assess the occurrence of malaria outbreak and investigate contracting factors of malaria in Simada District, Northwest Ethiopia. A single observation original research.

Results: Among the total 54 cases, 44 (81.5\%) of them were confirmed malaria cases. The average attack rate was 20 per 100 and slide positivity rate was $81.5 \%$. People in the age group of 5-14 years were most affected with an attack rate of $37 \%$. Presence of water bodies for mosquito breeding inside less than $1 \mathrm{~km}$ radius $(\mathrm{AOR}=3.32,95 \% \mathrm{Cl}$ 1.18-9.34), no knowledge on transmission, prevention and control mechanisms of malaria ( $A O R=4.36,95 \% \mathrm{Cl} 1.64$, 12.23), not using Insecticide Treated Bed Net (AOR $=5.85,95 \% \mathrm{Cl} 1.94,17.54)$ and absence of environmental control $(A O R=10.01,95 \% \mathrm{Cl} 2.94,33.33)$ were factors associated with malaria outbreak.
\end{abstract}

Keywords: Malaria, Outbreak, Investigation, Contracting factors, Case control, Simada, District, Ethiopia

\section{Introduction}

Malaria is endemic throughout the tropical areas of the world with the highest prevalence found in sub-Saharan Africa, India, and Southeast Asia [1]. There were about 219 million estimated malaria cases globally in 2017. About 200 million or $92 \%$ of malaria cases in 2017 were in the WHO African Region, followed by the WHO South-East Asia Region with 5\% of the cases [2]. In 2017, there were an estimated 435000 deaths from malaria globally. The WHO African Region accounted for $93 \%$ of all malaria deaths in 2017 [2].

In Ethiopia, about $68 \%$ of the total population resides in areas with high malaria risk [3] and 2,174,707 cases and 662 deaths due to malaria were reported in 20142015 with case fatality rate (CFR) of $0.03 \%$ [4]. There was, 1,127,241 malaria cases, out of a total population of 19,867,817 in Amhara Region in 2012 [5]. Low land areas (below 2000 meters of altitude) in Ethiopia are a place where more people are affected by malaria than high

\footnotetext{
*Correspondence: mekudesu@gmail.com

${ }^{2}$ Department of Human Nutrition, Institute of Public Health, College of Medicine and Health Sciences, University of Gondar, Gondar, Ethiopia Full list of author information is available at the end of the article
}

landers (above 2000 meters of altitude) [6]. The major malaria vector in Ethiopia is Anopheles arabiensis and the most dominant malaria parasites are Plasmodium falciparum (PF) and Plasmodium vivax (PV) [7]. Early diagnosis and prompt treatment, selective vector control, use of insecticide-treated mosquito nets (ITNs), and environmental management are the four main intervention strategies that are being applied in Amhara Region and Simada District, Workaye Kebele to combat malaria [8].

According to the Ethiopian national malaria indicator survey result $55.2 \%$ of households have at least one mosquito net and $38.2 \%$ of under five children had utilized ITNs [9]. In Amhara Region, 34.7\% and 16.6\% of households owned at least one net and one LLIN respectively. The mean numbers of nets and LLIN per house in Amhara Region were 0.5 and 0.3 respectively. Among those who had LLIN, only $12.5 \%$ were slept under LLIN or utilized it properly. From these who properly used LLIN, about $14.5 \%$ and $14.6 \%$ were under five children and pregnant women aged 15 to 49 years old respectively [10].

Population residing in malaria endemic areas of Amhara Region are affected during planting and harvesting seasons, cutting down productive capacity. It is also

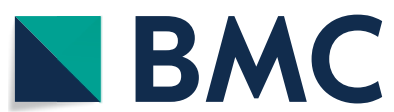

(c) The Author(s) 2019. This article is distributed under the terms of the Creative Commons Attribution 4.0 International License (http://creativecommons.org/licenses/by/4.0/), which permits unrestricted use, distribution, and reproduction in any medium, provided you give appropriate credit to the original author(s) and the source, provide a link to the Creative Commons license, and indicate if changes were made. The Creative Commons Public Domain Dedication waiver (http://creativecommons.org/ publicdomain/zero/1.0/) applies to the data made available in this article, unless otherwise stated. 
associated with loss of earnings, low school attendance, and high treatment costs and patient overload on health facilities $[4,11]$.

Therefore, this study aimed at investigating the causes of the outbreak in Simada District and identifying factors associated with contracting malaria. Besides, it also tried to describe outbreak trends by person place and time and thus providing feasible recommendations of the finding to control and preventive measures towards malaria outbreak.

\section{Main text \\ Methods}

The study was conducted in Workaye Kebele (the smallest administration unit in Ethiopia) from Jun 09 to 20/2016. Workaye is found in Simada District, Amhara region, Ethiopia. It is located $650 \mathrm{~km}$ far from Addis Ababa, the capital city of Ethiopia. The total population residing in the Kebele was estimated to be 7725 . Of which $51 \%$ were males and $49 \%$ were females. The study setting is located in dissected landscapes of Abay-Beshilo Basin of Simada District where land degradations, drought, food insecurity and famine are serious problems mainly since 1980s. It is totally included in the Abay River Basin. The elevations of the study area ranges from $854 \mathrm{~m}$ to $1500 \mathrm{~m}$ above sea-level. The temperature ranges from 24 to $28^{\circ} \mathrm{C}$ and rainfall from 200-900 mm (Fig. 1) [12].
On June 2016, a rumor of malaria outbreak in Workaye Kebele was notified to South Gondar Zone health department by Simada District health office. To verify the occurrence of the outbreak current data was compared with threshold. The 2016 data was compared with the average weekly number of cases during 20112015 to determine whether the epidemic threshold had been crossed.

Un-matched case-control study was used to identify risk factors of malaria outbreak from June 09 to 20, 2016. Data was collected by checklist on malaria outbreak risk factors in four Villages of Workaye Kebele. Selected cases and controls were interviewed and observation also was made on man mad water -holding bodies close to home and presence of anopheles' larvae in stagnant water.

Those people with and without malaria signs and symptoms for less than 2 weeks were selected from the community as cases and controls in 1:2 ratio. They were selected regardless of their age, gender, educational status, physiological status and socio-economic status. Epi Info version 3.5.1 and SPSS version 20 were used to describe the disease and analyze associated risk factors. $\mathrm{Bi}$-variable and multivariable analysis and Odds Ratio (OR) with its corresponding 95\% Confidence Interval (CI) were calculated to determine the significance of risk factors with the outbreak.

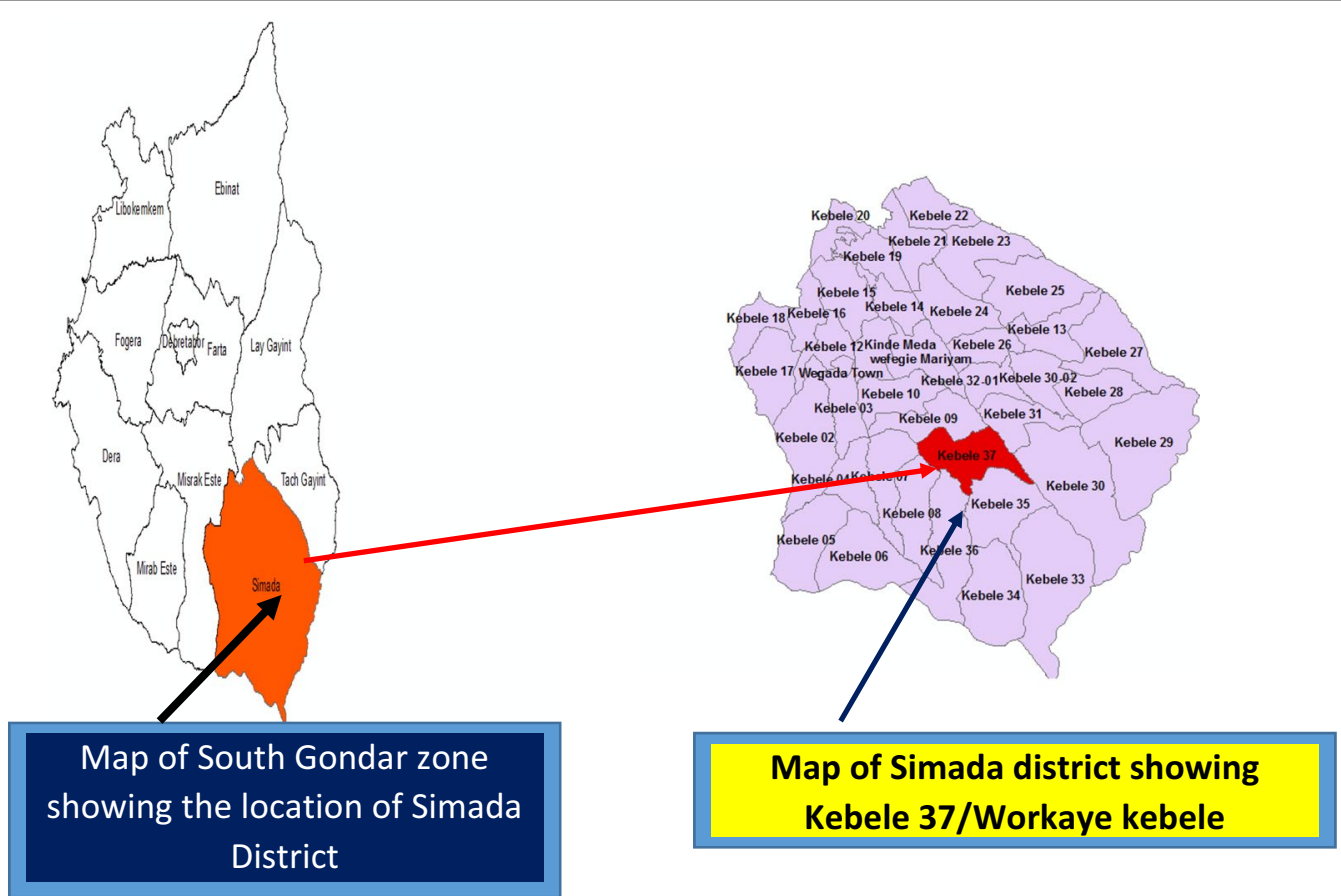

Fig. 1 Map of investigation area, Workaye Kebele, Simada District, Amhara Region, Ethiopia 2016 (source: Workaye Kebele administration office) 
The sample size was calculated using EPI-Info statistical package. The total calculated sample size was 162 (54 cases and 108 controls).

\section{Ethical considerations}

Ethical approval was obtained from Ethical review board of University of Gondar. Permission letter was also secured from Simada District health office. In addition, written consent was obtained from study participants after providing a clear information about the overall objectives of the study.

\section{Results}

\section{Socio demographic characteristics}

The study included $71(43.8 \%)$ males and 91 (56.2\%) females. Most of the participants were greater than 15 years. Concerning their ethnicity and religion all 162 (100\%) of them were Amhara and Orthodox Christian. Seventy-two participants (44.4\%) were married and 61 (37.7\%) were single (Table 1$)$.

\section{Descriptive epidemiology by person}

A total of 227 malaria cases were examined by RDT/ Microscopy. Of which 154 (67.8\%) were positive for malaria. Age group 5-14 years and females were the most affected groups with an attack rate of 37 and 29 per 100 population. The median age of cases and controls was 18 and 20 years. Average attack rate was 28 per 100 population with no malaria death. The positivity rate among randomly tested 50 fever cases was found to be $92 \%$ confirmed by slide microscopy which confirmed the existence of an outbreak according to the national guideline threshold of $>50 \%$ (Additional file 1: Table S1).

Due to the increased number of malaria cases from Workaye Kebele the threshold of Yekosa health center was crossed at WHO- Epi- Week 22 (Additional file 2: Figure S1).

Due to increased number of malaria cases from Welekoch, Tig mender, Edari mender and Addis Amba Villages, the threshold of Workaye health post was crossed at WHO- Epi- Week 22 (Additional file 3: Figure S2).

\section{Descriptive epidemiology by place}

Among the total population who were at risk of malaria, $280 / 835$ (33.5\%), 215/835 (25.8\%), and 155/835 (18.6\%) were from Addis Amba, Welekoch and Tig mender villages respectively. $P F$ followed by $P V$ were the most dominant species responsible for the outbreak. Positivity rate was higher in Addis Amba village (28.2\%) as compared to the rest. The attack rate was more in Tig mender and Welekoch Villages, 27 and 21 per 100 population (Additional file 4: Table S2).
Table 1 Socio demographic characteristics of the study participants in Workaye Kebele, Simada District, Northwest Ethiopia, 2016

\begin{tabular}{|c|c|c|c|c|}
\hline \multirow[t]{2}{*}{ Variable } & \multicolumn{2}{|c|}{ Cases } & \multicolumn{2}{|c|}{ Controls } \\
\hline & $\mathbf{N}$ & $\%$ & $\mathbf{N}$ & $\%$ \\
\hline \multicolumn{5}{|l|}{ Sex } \\
\hline$M$ & 28 & 51.9 & 43 & 39.8 \\
\hline $\mathrm{F}$ & 26 & 48.1 & 65 & 60.2 \\
\hline \multicolumn{5}{|l|}{ Marital status } \\
\hline Single & 32 & 59.3 & 29 & 26.9 \\
\hline Married & 15 & 27.8 & 57 & 52.8 \\
\hline Widowed/divorced & 7 & 13 & 22 & 20.3 \\
\hline \multicolumn{5}{|l|}{ Educational status } \\
\hline $\mathrm{N} / \mathrm{A}$ & 8 & 14.8 & 8 & 7.4 \\
\hline Unable to read and write & 11 & 20.4 & 36 & 33.3 \\
\hline Able to read and write & 8 & 14.8 & 20 & 18.5 \\
\hline Primary & 27 & 50 & 38 & 35.2 \\
\hline Secondary and above & 0 & 0 & 6 & 5.6 \\
\hline \multicolumn{5}{|l|}{ Ethnicity } \\
\hline Amhara & 54 & 100 & 108 & 100 \\
\hline Others & 0 & 0 & 0 & 0 \\
\hline \multicolumn{5}{|l|}{ Religion } \\
\hline Orthodox & 54 & 100 & 108 & 100 \\
\hline Others & 0 & 0 & 0 & 0 \\
\hline \multicolumn{5}{|l|}{ Occupation } \\
\hline $\mathrm{N} / \mathrm{A}$ & 10 & 18.5 & 9 & 8.3 \\
\hline Farmer & 16 & 29.6 & 63 & 58.3 \\
\hline Student & 28 & 51.9 & 36 & 33.3 \\
\hline
\end{tabular}

Laboratory Microscopy and RDT laboratory tests were done for 227 malaria cases in Yekosa health center and Welekoch health posts. Of the total tested cases, 154 (67.8\%) were positive for malaria. Among the positive cases, 104 (67.5\%) were $P V$ and 50 (32.5\%) $P F$ (Additional file 5: Figure S3).

\section{Analytical investigation}

In the Bi-vairiate logistic regression, factors that were significantly associated with increased risk of malaria case were traveling history with risk effect of significantly $(\mathrm{OR}=6.53$, CI2.19-19.5), presence of artificial water holding containers near to the home $(\mathrm{OR}=6.8$, CI3.314), presence of intermittent rivers with $1 \mathrm{~km}$ radius $(\mathrm{OR}=10, \mathrm{CI}$ 4.4-22.7) and staying outside the home overnight ( $\mathrm{OR}=4.6$, CI 2.16-9.78); whereas knowledge on transmission, prevention and control of malaria $(\mathrm{OR}=0.19$, CI $0.1-0.47)$, utilization of LLINs $(\mathrm{OR}=0.2$, CI 0.096-0.417) and environmental control $(\mathrm{OR}=0.22$, CI 0.1-0.473) were protective factors. While, presence of artificial water holding bodies near to the home, presence 
of intermittent rivers within $1 \mathrm{~km}$ radius, knowledge on malaria transmission, prevention and control, LLINs utilization, and environmental control were factors remained associated with malaria contracting during multivariate analysis.

Accordingly, people who were living in households where artificial water holding bodies were 3.3 times more at risk of contracting malaria than their counterparts $(\mathrm{AOR}=3.32,95 \%$ CI 1.18, 9.30). Likewise, presence of intermittent rivers closes to the community within $1 \mathrm{~km}$ distance increased the likelihood of getting malaria than those far away from it (AOR $=4.72,95 \%$ CI 1.6, 13.65).

Similarly, people who did not used ITNs were at higher risk of developing malaria than who used $(\mathrm{AOR}=5.85$, 95\% CI 1.94, 17.54). Furthermore, those who did not controlled their living environment were 10 times at higher risk of contracting the disease (AOR $=10.01,95 \%$ CI 2.94, 33.33) and higher odds of malaria were noted among those who had no knowledge on malaria transmission, prevention and control mechanisms $(\mathrm{AOR}=4.36,95 \% \mathrm{CI}$ 1.64, 12.23) (Table 2).

\section{Environmental assessment}

Mesk River and Kugena dam were found in Workaye Kebele nearest to affected Villages. As it was observed, there were multiple cracking sites favorable for mosquito breeding and mosquito larvae was seen on the river. Forty-two households from affected Villages were randomly selected and visited. According to the visit, water contained well, stagnant water and improper use of drainage system were observed. All households had at least one LLINs in their house. More than half (53.7\%) of them used LLINs and 32.5\% used it properly. Among those use did not used LLINs, most of them used it for other purposes, dirty, not properly hung at sleeping space and put under box. There were larvae of mosquitoes in observed stagnant water. According to clients' response, indoor residual spray was not performed for the last 1 year in all outbreak affected households.

\section{Interventions done}

- Focal spray was performed for all affected Villages.

- Dam on the river was opened.

- Education was given on prevention and control measures of malaria.

- Health professionals were assigned to affected Villages for early case management at community and health facility level.

- Mass fever treatment was given.

Table 2 Multivariate analysis of risk factors for malaria outbreak Workaye Kebele, Simada District, Northwest Ethiopia, 2016

\begin{tabular}{|c|c|c|c|c|c|c|c|c|c|}
\hline \multirow[t]{2}{*}{ Variables } & \multirow{2}{*}{$\begin{array}{l}\text { Cases } \\
\mathrm{N}=50(\%)\end{array}$} & \multirow{2}{*}{$\begin{array}{l}\text { Controls } \\
\mathrm{N}=100(\%)\end{array}$} & \multirow[t]{2}{*}{ COR } & \multicolumn{2}{|l|}{$95 \% \mathrm{Cl}$} & \multirow[t]{2}{*}{ AOR } & \multicolumn{2}{|l|}{$95 \% \mathrm{Cl}$} & \multirow[t]{2}{*}{$P$ value } \\
\hline & & & & Lower & Upper & & Lower & Upper & \\
\hline \multicolumn{10}{|c|}{ Traveling history } \\
\hline Yes & 13 & 5 & 6.53 & 2.19 & 19.5 & \multirow{2}{*}{$\begin{array}{l}2.21 \\
1\end{array}$} & \multirow[t]{2}{*}{0.84} & \multirow[t]{2}{*}{3.86} & \multirow[t]{2}{*}{0.12} \\
\hline No & 41 & 103 & 1 & & & & & & \\
\hline \multicolumn{10}{|c|}{ Presence of artificial water holding bodies near to the home } \\
\hline Yes & 35 & 23 & 10 & 4.4 & 22.7 & \multirow{2}{*}{$\begin{array}{l}3.32 \\
1\end{array}$} & \multirow[t]{2}{*}{1.18} & \multirow[t]{2}{*}{9.3} & \multirow[t]{2}{*}{$<0.001$} \\
\hline No & 19 & 85 & 1 & & & & & & \\
\hline \multicolumn{10}{|c|}{ Presence of intermittent rivers with $1 \mathrm{~km}$ radius } \\
\hline Yes & 45 & 36 & 5.41 & 2.11 & 10.00 & \multirow{2}{*}{$\begin{array}{l}4.72 \\
1\end{array}$} & \multirow[t]{2}{*}{1.6} & \multirow[t]{2}{*}{13.65} & \multirow[t]{2}{*}{0.004} \\
\hline No & 9 & 72 & 1 & & & & & & \\
\hline \multicolumn{10}{|c|}{ Knowledge on transmission, prevention and control of malaria } \\
\hline Yes & 17 & 81 & 1 & & & \multirow{2}{*}{$\begin{array}{l}1 \\
4.36\end{array}$} & \multirow{2}{*}{1.64} & \multirow[t]{2}{*}{12.23} & \multirow[t]{2}{*}{0.005} \\
\hline No & 37 & 27 & 5.00 & 2.40 & 10.42 & & & & \\
\hline \multicolumn{10}{|c|}{ Staying outside the home overnight } \\
\hline Yes & 24 & 16 & 6.8 & 3.3 & 14 & \multirow{2}{*}{$\begin{array}{l}3.23 \\
1\end{array}$} & \multirow[t]{2}{*}{0.78} & \multirow[t]{2}{*}{4.32} & \multirow[t]{2}{*}{0.07} \\
\hline No & 30 & 92 & 1 & & & & & & \\
\hline Utilization & & & & & & & & & \\
\hline Yes & 27 & 90 & 1 & & & 1 & 1.94 & 17.54 & 0.002 \\
\hline No & 27 & 18 & 4.61 & 2.16 & 9.78 & 5.85 & & & \\
\hline Environme & ontrol & & & & & & & & \\
\hline Yes & 11 & 58 & 1 & & & 1 & 2.94 & 33.33 & $<0.001$ \\
\hline No & 43 & 50 & 4.54 & 2.11 & 10.00 & 10.01 & & & \\
\hline
\end{tabular}


- Contributing factors for malaria outbreak were rapidly assessed in the environment and control of breeding sites was done.

- Surveillance system was strengthened.

\section{Discussion}

Based on 5 years' epidemiological records of malaria cases, the study findings confirmed existence of malaria epidemic in the study area. Malaria outbreak began in the area was in line with other areas of Ethiopia and the national data in terms of time period when increased malaria cases reported. April to December is a period in which malaria epidemic is commonly happened in different regions of Ethiopia [13].

The number of malaria cases reported were doubled compared to the prior year in WHO epidemic week $22 / 2016$. The peak magnitude of malaria cases showed in week 26/2016. Index case from Epi-curve and threshold graph showed outbreak response was started late.

Age group of 5-14 years and females were more affected with AR of 37 and 29 per 100 population. Studies in India [14], Ethiopia [15] and Zimbabwe [16] showed children and females were more attacked by malaria. The reason may be children have lower immunity and adult women do more activities that exposed them to mosquito breeding sites.

Households found within $1 \mathrm{~km}$ radius had odds of 4.72 of contracting malaria as compared to those far away. This is due to presence of multiple cracked sites of Mesk River and was favorable for mosquito breeding. A study in Zimbabwe reported the association of staying close to such water sources and contracting malaria [16]. Hence, there should be sustainable integrated and coordinated malaria prevention and control measures and proper water management to eliminate mosquito breeding sites [17].

Stagnant water formed following heavy rainfall creates a conducive breeding site for mosquitos and is a cause for malaria epidemics [18]. Similarly, our study recognized that people found close to the stagnant water were 3.3 times more affected than those who lived far from it. This finding is supported by a report from Afar, Ethiopia [19].

A study in Kenya showed household ownership of a mosquito net was associated with lower risk of malaria impact and transmission [20]. Studies from Zimbabwe and Beitbridge reported that not having an ITN hanged in the room was significantly associated with contracting Malaria [16, 21]. Findings of this study also revealed similar results.

Awareness about malaria is an important aspect to prevent disease exposure and its related negative health consequences [22, 23]. A study in Ethiopia revealed that general knowledge about malaria helped the community to prevent and control the disease thereby, it has a significant contribution in the reduction of burden of malaria [24]. Similarly, in the current study, having no awareness on means of transmission and prevention and control measures of malaria was a risk of contracting the disease.

The likelihood of occurrence of the outbreak in the current study area increased tenfolds in poorly controlled environment. Similar study finding was also reported from other part of Ethiopia [15].

\section{Conclusion}

Malaria outbreak was confirmed in Workaye Kebele. Age group of 5-14 years and females were more affected by malaria outbreak. Lack of awareness on malaria transmission and control, presence of rivers, dams, stagnant water, poor bed net utilization, presence of artificial water bodies and poor environmental control were significantly associated with the occurrence of malaria outbreak.

Therefore, community awareness regarding ITN utilization and malaria prevention and control mechanism should be created. Beside, utilization of bed net should be monitored and optimized. Regular indoor residual spray should have to be done before rainy season twice per year. Removal of potential mosquito breeding sites should be conducted regularly.

\section{Limitations of the study}

Knowledge of study population regarding importance, duration, and care of insecticide treated bed nets and availability of chemical spray in the District health office stock and the reason why indoor chemical spray were not assessed.

\section{Additional files}

Additional file 1: Table S1. Malaria Attack Rate per 100 population by age and sex in Workaye Kebele, Simada District, Northwest Ethiopia. This data shows which gender and age group is more affected among the total population in Wokaye Kebele (malaria attack rate per 100 population with respect to age and sex).

Additional file 2: Figure S1. Threshold of Malaria in Yekosa health center in Workaye Kebele, Simada District, Northwest Ethiopia. It shows number of malaria cases in Yekosa health center in comparison to WHO week and 2015 (WHO week that showed malaria threshold comparing, cases of 2015 with 2016)

Additional file 3: Figure S2. Malaria cases threshold in Workaye health post, Workaye Kebele, Simada District, Northwest Ethiopia. It shows number of malaria cases in Workaye health post in comparison to WHO week and 2015 (WHO week that showed malaria threshold comparing, cases of 2015 with 2016)

Additional file 4: Table S2. Malaria attack rate by Village in Workaye Kebele, Simada District, Northwest Ethiopia. This data is about the distribution of malaria across affected Villages in Workaye Kebele (distribution of total malaria cases in four Villages and attack rate per 100 population among the total population). 
Additional file 5: Figure S3. Epidemic curve of malaria outbreak in Workaye Kebele, Simada District, Northwest Ethiopia. This data provides an information about the increment of malaria cases as compared to the previous number of cases, the time when the index case was identified, date of onset, notification time to Zonal public health emergency management and the time when the investigation was started.

\section{Abbreviations}

AOR: adjusted odd ratio; AR: attack rate; CFR: case fatality rate; $\mathrm{Cl}$ : confidence interval; COR: crude odd ratio; IRS: indoor residual spray; ITN: insecticidetreated Mosquito Net; KM: kilo meter; LLIN: long-lasting insecticide-treated nets; OR: odd ratio; PF: Plasmodium falciparum; PHEM: Public Health Emergency Management; PV: Plasmodium vivax; RDT: Rapid Diagnostic Test; RRT : Rapid Response Team; SPSS: Statistical Package for Social Sciences; WHO: World Health Organization.

\section{Acknowledgements}

Authors would like to thank South Gondar Zone health department, Simada health office and Yekosa health center for providing the necessary support and information during the study period. We also like to thank study participants and data collectors for their valuable time and devotion.

\section{Authors' contributions}

BW: Research idea generation, research tool development, data analysis and report write up, FAM: participated in protocol development, data collection and analysis, KAG: involved in data collection, report write up and revised the manuscript, MS: data analysis, report writing and manuscript preparation. All authors read and approved the final manuscript.

\section{Funding}

No funding was received for this work.

\section{Availability of data and materials}

Data are available from the corresponding author on reasonable requests.

\section{Ethics approval and consent to participate}

Ethical approval for this work was obtained from Ethical review board of University of Gondar. Permission letter was also secured from South Gondar Zonal health department and Simada District health office. In addition, written consent was obtained from study participants after providing a clear information about the overall objectives of the study.

\section{Consent for publication}

Not applicable.

\section{Competing interests}

The authors declare that they have no competing interests.

\section{Author details}

1 Department of Epidemiology and Biostatistics, Institute of Public Health, College of Medicine and Health Sciences, University of Gondar, Gondar, Ethiopia. 2 Department of Human Nutrition, Institute of Public Health, College of Medicine and Health Sciences, University of Gondar, Gondar, Ethiopia.

Received: 8 March 2019 Accepted: 14 May 2019

Published online: 17 May 2019

\section{References}

1. Kansas Department of Health and Environment Investigation Guidelines. Malaria Disease Management and Investigative Guidelines. 2018.

2. World malaria report 2018. World Health Organization 2018. ISBN 978-92-4-156565-3.

3. President Malaria Initiative. Ethiopia Malaria Operation Plan FY 2015. https ://www.pmi.gov/docs/default-source/default-document-library/malar ia-operational-plans/fy-15/fy-2015-ethiopia-malaria-operational-plan. pdf?sfvrsn $=3$.

4. Health Sector Development Programme Iv, Annual Performance Report. Ministry of Health of Ethiopia. 2015.

5. Ayalew S, Mamo H, Animut A, Erko B. Assessment of current malaria status in light of the ongoing control interventions, socio-demographic and environmental variables in Jiga Area, Northwest Ethiopia. PLoS ONE. 2016;11:e0146214.

6. National Strategic Plan for Malaria Prevention, Control And Elimination in Ethiopia: 2011-2015.

7. Ministry of Health of Federal Democratic Republic of Ethiopia. National malaria guidelines 3rd edition. 2012

8. Alemu A, Muluye D, Mihret M, Adugna M, Gebeyaw M. Ten year trend analysis of malaria prevalence in Kola Diba, North Gondar, Northwest Ethiopia. Parasit Vectors. 2012;5:173.

9. Federal Democratic Republic of Ethiopia, Ministry of Health. Ethiopia National Malaria Indicator Survey 2011: Technical Summary. Addis Ababa: The Ethiopian Health and Nutrition Research Institute \& Partners; 2012.

10. Prevalence and risk factors for malaria and trachoma in ethiopia. A household cluster survey of malaria prevalence and risk factors in Amhara, Oromia and southern nations, nationalities and peoples' regions, trachoma prevalence and risk factors in Amhara region. August, 2007.

11. Adugna A. Malaria in Ethiopia, Lesson 14, www.ethiodemographyandhe alth.org/MedVectoredDiseasesMalaria.

12. Menberu Teshome. Agricultural Susceptibility to Climate Change in Varied Ecological areas of Northwest Ethiopia. American Journal of Agricultural Research (ISSN:2475-2002). AJAR (2017), 2:6.

13. The Intersectoral response to the malaria epidemic in Ethiopia in 2003: an assessment, Yolanda Barbera Lainez, International Rescue Committee.

14. Rana RK, Kumar R, Kashyap V, Karn PK, Soren SK. A report on outbreak investigation of malaria in Gaya district, Bihar, India establishing new niches. Int J Med Health Res. 2016;2(12):01-8.

15. Lake MW, Mebratur M, Mehari D, Dessie K. Epidemiological analysis of malaria outbreak in Ankesha District, Awi Zone, Amhara region, Ethiopia, 2012: weaknesses in control measures and risk factors. Sci J Public Health. 2016:4(2):132-7.

16. Kureya T, Ndaimani A, Mhlanga M. Malaria Outbreak Investigation in Chipinge, Zimbabwe: a case-control study. Iran J Parasitol. 2017;12(3):423-32.

17. Jennifer $K$, Jürg $U$, Marcel T. The effect of irrigation and large dams on the burden of malaria on global and regional scale. Trop Med Hyg. 2005;72(4):392-406.

18. Dawit. G. Prevalence and risk factors of malaria in Ethiopia; 2012.

19. Debela MB, Kahsay AB, Mokonnon TM. Malaria outbreak and contracting factors in Afar Region, Ethiopia. J Public Health Epidemiol. 2018;10(7):233-40.

20. Atieli. H. ITN usage in Kenya. 2011. journal Med.

21. Chiruvu RT, Kanengoni B, Mungati M, et al. Malaria Outbreak investigation in Chitulipasi, Beitbridge District, Matabeleland South Province, 2015. J Health Sci Nurs. 2017;2(5):104-20.

22. Yimer F, Animut A, Erko B, Mamo H. Past five year trend, current prevalence and household knowledge, attitude and practice of malaria in Abeshge, South central Ethiopia. Malar J. 2015;14:230.

23. Erhun WO, Agbani EO, Adesanya SO. Malaria prevention: knowledge, attitude and practice in a Southwestern Nigerian community. Afr J Biomed Res. 2006;8:25-9.

24. Bedimo H, Fikrie N, Hailu A. Knowledge, attitude and practice on malaria and associated factors among residents in Pawe district, north west Ethiopia: a cross-sectional study. Sci J Public Health. 2015;3:3.

\section{Publisher's Note}

Springer Nature remains neutral with regard to jurisdictional claims in published maps and institutional affiliations. 